\title{
Annotation \\ Developmental Correlations of the Deciduous and Permanent Teeth During the Human Fetal Period
}

\author{
ALPHONSE R. BURDI and JEROME SUPERSTINE \\ Department of Anatomy (Medical School) and School of Dentistry, University of Michigan, \\ Ann Arbor, Michigan 48109, USA
}

J Dent Res 56(12): 1468, December 1977.

Early prenatal development of human dentitions through the classical stages of tooth development can be variable. Recognizing that there are patterns of typical and polymorphic tooth germ maturation for the deciduous and permanent dental arches during the human embryonic and fetal periods, this investigation was undertaken to examine correlations bet ween the deciduous and permanent teeth and their histogenesis during the critical morphogenic periods before birth.

Twenty-six human abortuses representing tooth development from dental bud through early apposition stages were used. The sample, ranging from 12-28 weeks fertilization age $(75.280 \mathrm{~mm}$ crown-rump length) was selected on the bases of being typical for age, no gross signs of abnormal development, and no remarkable clinical histories. Each specimen was serially sectioned in the sagittal plane and prepared for light microscopy (trichrome connective tissue stain). The developmental status of each available deciduous and permanent tooth of each specimen was assessed.

Maturational data were grouped and correlated statistically on the bases of tooth type, tooth positions, and jaw location, demonstrating (1) the relationship of any two dental variables over the crown-rump length range, and (2) the actual relationship of any two dental variables that might be expected when crown-rump length is constant.

Several patterns are discernible in deciduous and permanent tooth germ prenatal maturation. There is a significant correlation ( 0.01 level) in the progressive maturation of each tooth of an opponent pair (Fig 1). Holding age or crown-rump length constant through partial correlations $\left(r_{C R L}\right)$, this correlation holds true berween opponent tooth germs in both deciduous and permanent dentitions. The high correlations in tooth development also are shown by pooling data from all available deciduous $\left(r=0.97 ; r_{C R L}=0.89\right)$ and per manent $\left(r=0.91 ; r_{C R L}=0.80\right)$ tooth germs. A different pattern can be shown for the correlative development of adjacent teeth in the deciduous arch (central incisor through second molar) and the two teeth of the permanent arch (central and lateral incisors) which were monitored for adjacency. Raw order correlations ( $r$ ) indicate that adjacent teeth of either arch (deciduous 0.93 ; permanent 0.66 ) are significantly correlated in their development over the three to seven month period. With the exception of adjacent permanent incisors $\left(\mathrm{r}_{\mathrm{CRl}}=0.23\right)$, there is still a high correlation between adjacent deciduous teeth $\left(\mathrm{r}_{\mathrm{CR}}\right.$. $=0.80$ ) when crown-rump length is held constant by partial correlation procedures; although partial correlation values are lower throughout. In terms of correspondence in development between like teeth of the

Received lor publication June 6, 1977.

Accepted for publication July $7,1977$.

This research was supported in part by the Nutionat fustizute of Dental Research. NIH, Research Gran R01 DE-03443-06. and by the Beatrice Stanton Medical Research Fund. The University of Michigan. deciduous and permanent dental arches, raw order correlations indicate a moderate association ( $\mathrm{r}=$ 0.68 ) between like deciduous and permanent teeth during this time period. However, partial correlations show no significant association $\left(\mathrm{r}_{\mathrm{CRL}}=0.23\right.$ ) between the dentitions.

A demonstrable correlation was shown in development of opponent, adjacent, and even corresponding teeth in and between the deciduous and permanent dental arches during prenatal months 3 to 7 . Partial correlation procedures show, however, that without the effects of increasing age and/or crown-rump length on tooth development there is asynchronous or independent maturation of those deciduous and permanent teeth monitored in the same individual. Procedurally, the different messages provided by use of raw order and partial correlations argues for this kind of statistical model when analyzing morphologic traits with or without reference to increasing age.

The finding of developmental independence between deciduous and permanent tooth development then raises several queries, including to what degree can the development of prenatal deciduous teeth be altered, e.g., through teratogens, without a concurrent alteration in the typical maturation pattern of the permanent teeth? Similarly, since there have generally been strong correlations between maxillary and mandibular teeth of both deciduous and permanent tooth types during this prenatal period, to what ex. tent does altered embryonic development of teeth in one jaw affect teeth in the opponent jaw?

\section{DECIDUOUS TEETH}

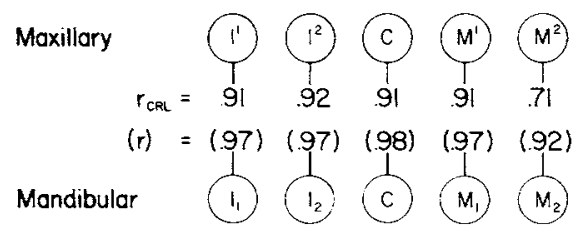

\section{PERMANENT TEETH}

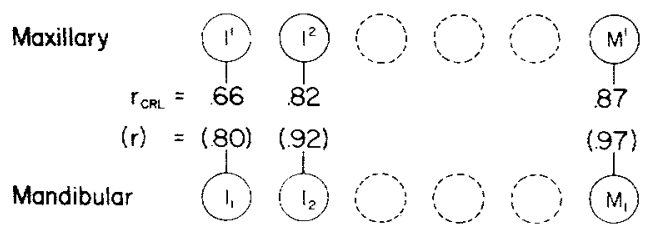

Developmental association between opponent looth germs of the deciduous and permanent arches as shown by raw order (r) and partial (r.RI) ) correlations. 